\title{
Obtaining Femtoscopy Results in Models with Resonances
}

\author{
Adam Kisiel \\ Faculty of Physics, Warsaw University of Technology, ul. Koszykowa 75, 00-662 Warsaw, Poland
}

Received on 23 November, 2006; revised version received on 16 March, 2007

\begin{abstract}
We present femtoscopic results from models in which the resonance contribution is taken into account. The Therminator program, which implements a single freeze-out model and takes into account propagation and decay of all known resonances, was used. We test methods of determining the femtoscopic size of the system, or the "HBT radii". We show that the best one is the two-particle method combined with the exact calculation of the two-pion wave function including the wave-function symmetrization and Coulomb effects. We compare it to other methods and comment on their validity and applicability.
\end{abstract}

Keywords: Femtoscopy; Resonance propagation; HBT radii

\section{FEMTOSCOPIC DEFINITIONS}

The femtoscopy measurements have been extensively used to study the heavy-ion collisions. For recent review of theoretical and experimental results please see [1]. Femtoscopic correlation function is normally constructed as:

$$
C(\vec{q}, \vec{K})=\frac{P_{2}^{C}(\vec{q}, \vec{K})}{P_{2}^{0}(\vec{q}, \vec{K})}
$$

where $P_{2}^{C}$ is the probability to observe two femtoscopically correlated particles at relative momentum $\vec{q}$. $P_{2}^{0}$ is such probability where the correlation between particles does not have the femtoscopic component. $\vec{K}$ is the average momentum of the pair. In heavy-ion experiments the $P_{2}^{C}$ is usually constructed from pairs coming from the same event; $P_{2}^{0}$ from pairs where each particle comes from different event, but the events are as close to each other in global characteristics as possible.

In theoretical models one should, in principle, generate particles in such a way that they are already correlated due to their mutual and many-particle interactions. That is however usually computationally not possible. One then makes an assumption that the interaction between particles can be separated from their generation and we write the most general form of the correlation function that can be used by models:

$$
C(\vec{q}, \vec{K})=\frac{\int S\left(\mathbf{r}^{*}, \vec{q}, \vec{K}\right)\left|\Psi\left(\vec{q}, \mathbf{r}^{*}\right)\right|^{2} d^{4} \mathbf{r}^{*}}{\int S\left(\mathbf{r}^{*}, \vec{q}, \vec{K}\right) d^{4} \mathbf{r}^{*}}
$$

where $\mathbf{r}^{*}$ is the pair separation in the pair rest frame (PRF) and $S\left(\mathbf{r}^{*}, \vec{q}, \vec{K}\right)$ is the pair separation distribution constructed as:

$S\left(\mathbf{r}^{*}, \vec{q}, \vec{K}\right)=\int S_{1}\left(\mathbf{x}_{1}, \vec{p}_{1}\right) S_{2}\left(\mathbf{r}^{*}-\mathbf{x}_{2}, \vec{p}_{2}\right) \boldsymbol{\delta}\left(\mathbf{r}^{*}-\mathbf{x}_{1}+\mathbf{x}_{2}\right) d^{4} \mathbf{x}_{1} d^{4} \mathbf{x}_{2}$

and $S(\mathbf{x}, \vec{p})$ is the single-particle emission function provided by the model. For identical particles $S_{1} \equiv S_{2}$ and $S\left(\mathbf{r}^{*}\right)$ is symmetric by definition, for non-identical particles $S\left(\mathbf{r}^{*}\right)$ is usually asymmetric. In this work we only consider identical particles, more specifically charged pions. If one takes into account the proper symmetrization of the wave function only:

$$
\left|\Psi\left(\vec{q}, \mathbf{r}^{*}\right)^{Q}\right|^{2}=1+\cos \left(\vec{q}^{*}\right)
$$

one obtains a quantum-statistics only correlation function $C^{Q}$ which has a simple form and is useful in model studies. One can also consider Coulomb interaction:

$$
\begin{aligned}
\left|\Psi\left(\vec{q}, \mathbf{r}^{*}\right)^{Q C}\right|^{2} & =\mid \frac{\sqrt{A_{C}}}{\sqrt{2}}\left[\exp \left(-i \vec{k}^{*} \vec{r}^{*}\right) F\left(-i \eta, 1, i \xi^{+}\right)\right. \\
& \left.+\exp \left(\vec{k}^{*} \vec{r}^{*}\right) F\left(-i \eta, 1, i \xi^{-}\right)\right]\left.\right|^{2}
\end{aligned}
$$

where $k^{*}$ is half of pair relative momentum in PRF, $A_{C}$ is the Gamow factor, $F$ is the confluent hypergeometric function, $\eta=1 / k^{*} a_{c}, a_{c}$ is the pair Bohr radius and $\xi^{ \pm}=k^{*} r^{*} \pm \vec{k}^{*} \vec{r}^{*}$. Then one obtains the full correlation function that can be compared to data.

It is possible to use Eq. 2 to get the model correlation function. However it is often difficult to perform the complete integration, so various approximations are used, which will be presented in the next sections. Another possibility is to perform the integral numerically. One needs to generate particles according to the single-particle emission function. Then one combines the particles into pairs and creates two histograms in one of them the squared wave-function of the pair is stored, in the other: unity for each pair. The result of the division of the two histograms is the average pair wave function in each bin, which is the correlation function per Eq. (2).

\section{OBTAINING HBT RADII}

Femtoscopic analysis provides information about the size of the system in terms of the "HBT radii". It is also possible to obtain information about the separation distribution in a model independent way using the "imaging" technique $[2,3]$. The size of the system can be determined separately in three directions: "out" - along the pair momentum, "long" - along the beam axis and "side" - perpendicular to the other two. All analysis are performed in LCMS system, which is defined as the one in which pair momentum along the beam axis vanishes. The radii are obtained under the following assumptions. The emission function is static (does not depend on particle's momentum) and is a $3 \mathrm{~d}$ sphere with gaussian density profile:

$$
S(\mathbf{r}) \approx \exp \left(\frac{-r_{\text {out }}^{2}}{4 R_{\text {out }}^{2}}+\frac{-r_{\text {side }}^{2}}{4 R_{\text {side }}^{2}}+\frac{-r_{\text {long }}^{2}}{4 R_{\text {long }}^{2}}\right)
$$


The correlation function is then:

$$
C(\vec{q})=1+\lambda \exp \left(-R_{\text {out }}^{2} q_{\text {out }}^{2}-R_{\text {side }}^{2} q_{\text {side }}^{2}-R_{\text {long }}^{2} q_{\text {long }}^{2}\right)
$$

and can be easily fit to the experimental one to obtain the "HBT radii" $R_{\text {out }}, R_{\text {side }}$ and $R_{\text {long. }}$.

Two other methods of obtaining the radii have been tested. They involve more assumptions, but are simpler to perform analytically. We will test their validity.

The distribution in Eq. (7) is a convolution of two singleparticle distributions (see Eq. (3)). It can approximated by the square of them:

$$
S(\mathbf{r}) \approx S(\vec{x}, \vec{p})^{2}
$$

where $\vec{p}$ is substituted for $\vec{K}$. This is rigorously true only if $S(x, p)$ is a static Gaussian. For any realistic $S$ it is only an approximation, the validity of which is under study. Using Eq. (9) and Eq. (4) one can write the following approximate formula for the correlation function, known as the "Wigner formalism":

$$
C(\vec{q})=\frac{\left|\int S(\vec{x}, \vec{p}) e^{i \vec{q} \vec{x}} d^{3} x\right|^{2}}{\left|\int S(\vec{x}, \vec{p}) d^{3} x\right|^{2}}
$$

This function can again be fit by Eq. (8) to obtain the "HBT radii". The advantage of this procedure is that it can be performed analytically using only the single-particle emission function. An example of the correlation function calculated in this way is shown in Fig. 1, together with the reference function from the two-particle method.

An even simpler procedure is to analyze the emission function $S$ directly. One can either obtain its Gaussian width or calculate the relevant RMS'es. One can do it to single particle emission function $S(\vec{x})$, which is easier to do, but involves additional approximation, or to the two particle emission function $S(\vec{r})$. For the exact formulas used in the calculation of RMS'es of $S(\vec{x})$ please consult [4]. Again these methods will give results identical to the previous ones only if the emission functions are Gaussian. The validity of the approximations was tested, the results are discussed in the next paragraph.

\section{METHOD OF TESTING}

We test and compare various methods to obtain the "HBT radii". We treat the two-particle method with the wavefunction calculation as the reference, or the method which most closely resembles the experimental one, to which it should be compared.

The input data were the events generated with the THERMINATOR [5] which takes into account all the resonances from the PDG [6]. It uses the Blast-wave-like model with parameters fit to RHIC central data and a freeze-out hypersurface with negative slope in $\rho-t$ plane (outside-in freezeout). The two-particle method, described in detail in [7], was used to get the reference radii. The correlation functions according to (10) were calculated using the same events and a Monte-Carlo integration on a 3-dimensional mesh in $\vec{q}$ space.
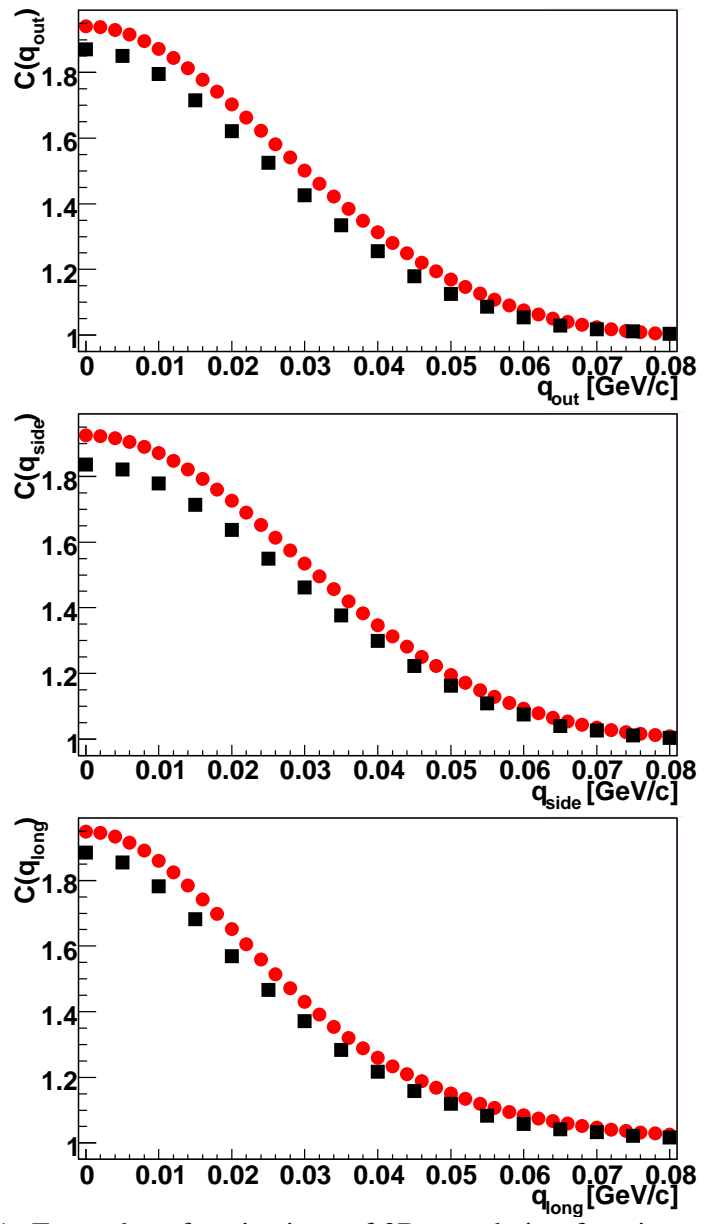

FIG. 1: Examples of projections of 3D correlation functions. From top to bottom: $C\left(q_{\text {out }}\right), C\left(q_{\text {side }}\right)$ and $C\left(q_{\text {long }}\right)$, with other components of $q$ integrated over the range of $10 \mathrm{MeV}$. Black squares are the correlation function from the reference two-particle method. Red circles are the correlation function calculated using the Wigner formalism.

The corresponding variances were also calculated, according to formulas given for HBT radii in [4].

In order to test the validity of each method the following test was done. Therminator model generates all particles at the freeze-out hypersurface. The unstable ones then decay strongly in cascades until only stable particles remain. Inclusion of resonances is known to produce long-range, nongaussian tails in the separation distributions. Such situation is expected to occur in measured events, and therefore is also a very good "real-world" test of the approximate HBT radii determination methods, which rely on the gaussianness of the distributions. Long-range $(r>50 \mathrm{fm})$ tails are not expected to significantly influence the radius parameter obtained in the femtoscopic analysis. They influence lambda parameter only. One can determine the "HBT radii" as a function of the cut-off of the emission time of the particles taken into account. It is a test of the stability of the method. The expected behavior is that above some limiting cut-off value the radii should remain stable and only the $\lambda$ parameter should change. 

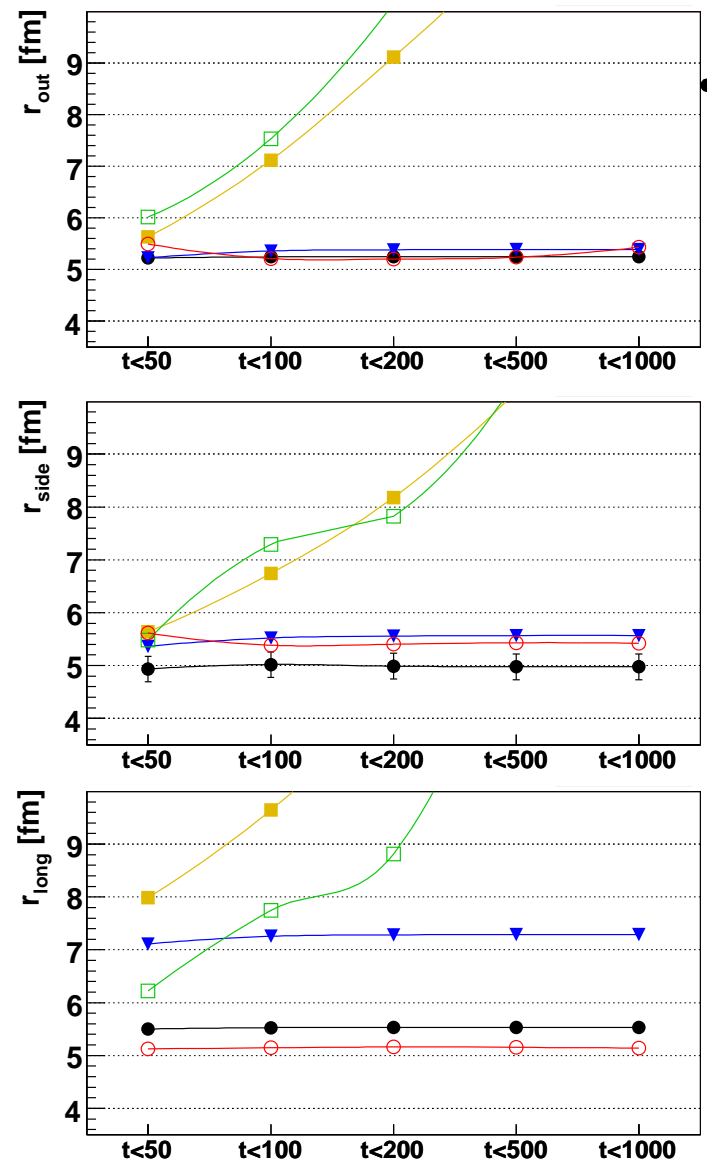

FIG. 2: "HBT radii" as a function of the maximum emission time cut-off. From top to bottom: $r_{\text {out }}, r_{\text {side }}$ and $r_{\text {long }}$. Black filled circles are the reference two-particle method. Filled yellow squares RMS of $S(\vec{r})$, blue triangles - $\sigma$ of Gaussian fit to $S(\vec{r})$, open green squares - RMS of $S(\vec{x})$, open red circles: "HBT radii" from the fit to single particle (calculated using the Wigner formalism) correlation function.

\section{RESULTS AND DISCUSSION}

The main result of this study is shown in Fig. 2. Several conclusions can be drawn. First, any measure based on the RMS of $S(\vec{x})$ or $S(\vec{r})$ is not a good approximation of the "HBT radii". Including strongly decaying resonances makes them go to very large values. It is to be expected as the particles which decay later, travel some distance before their decay, creating long-range tail in the distributions, to which RMS'es are extremely sensitive. This sensitivity to tails is not present in the radii obtained from the correlation function, and therefore is the weakness of the RMS method.

The next method is the Gaussian $\sigma$ of $S(\vec{r})$. It has the desired stability versus the time cut-off. However it still can differ from the reference method by up to $30 \%$. Moreover the fitted radius is dependent on the fit range taken. Also this method does not give any reasonable estimate of the $\lambda$ value. Therefore it can be used only as a crude estimate of the femtoscopic radii.

The last method compared is the fit to the correlation function calculated using the Wigner formalism (10). It is stable versus the time cut-off. It shows very good agreement with the reference method for the "out" radius, but the other two can differ up to $10 \%$. It does not permit to calculate the correlation function in an arbitrary reference frame, as the twoparticle method does. The Coulomb interaction cannot be introduced at all, as it can be done only for a pair of particles. A direct comparison of the correlation functions calculated in this way to the reference is shown in Fig. 1. The widths of the functions are comparable, which is also reflected in the radii. However the two-particle function shows lower intercept parameter. In other words the $\lambda$ is overestimated in the Wigner method. The reason is the following. Particles at large $r$ are effectively uncorrelated, and thus lower the $\lambda$. So for singleparticle emission function we have:

$$
\lambda=\frac{N_{s}}{N_{l}+N_{s}}=\frac{1-N_{l}}{N_{l}+N_{s}}
$$

where $N_{l}$ is the number of particles at large $r$ and $N_{s}$ are the particles at small $r$ (in the source). But a pair is uncorrelated even if only one particle in it is from large $r$, therefore $\lambda$ is:

$$
\lambda=\frac{N_{s}^{2}}{\left(N_{l}+N_{s}\right)^{2}}=\frac{1-N_{l}^{2}-2 N_{l} N_{s}}{\left(N_{l}+N_{s}\right)^{2}}
$$

which is smaller than (11) and is the correct value. Therefore the Wigner method can be used when the accuracy of $10 \%$ in the radii is acceptable. Also it cannot be used in more advances studies, for example in the direct comparisons of the correlation functions between theory and experiment.
[1] M. A. Lisa, S. Pratt, R. Soltz, and U. Wiedemann, Ann. Rev. Nucl. Part. Sci. 55, 357 (2005) [arXiv:nucl-ex/0505014].

[2] D. A. Brown and P. Danielewicz, Phys. Lett. B 398, 252 (1997) [arXiv:nucl-th/9701010].

[3] D. A. Brown, A. Enokizono, M. Heffner, R. Soltz, P. Danielewicz, and S. Pratt, Phys. Rev. C 72, 054902 (2005) [arXiv:nucl-th/0507015].

[4] F. Retiere and M. A. Lisa, Phys. Rev. C 70, 044907 (2004)
[arXiv:nucl-th/0312024].

[5] A. Kisiel, T. Taluc, W. Broniowski, and W. Florkowski, Comput. Phys. Commun. 174, 669 (2006) [arXiv:nucl-th/0504047].

[6] S. Eidelman, et al., Phys. Lett. B 592, 1 (2004)

[7] A. Kisiel, W. Florkowski, and W. Broniowski, Phys. Rev. C 73, 064902 (2006) [arXiv:nucl-th/0602039]. 